\title{
Highly efficient modelling of electronic structure
}

Chemistry can be defined as the study of electron motion in the field of nuclei, which obeys the rules of quantum structure of a given system structure of a given system of by an $N$-electron wave function built up with one-electron wave functions (orbitals) subject to the antisymmetry principle. The major issue is that the orbitals may be strongly entangled so as to render the $\mathrm{N}$-electron wave function extremely complicated and dh of th truncate - the function grows in combination with the numbers of electrons and orbitals. Professor Wenjian Liu (Shandong University, China) and collaborators have developed a highly efficient quantum chemical method which better accounts for electron correlation while keeping the resources relatively low. n chemistry, the smallest unit that makes
up all matter in the world - all that we
touch, see, and breathe - is the atom. As we understand it today, an atom consists of a nucleus made of protons and neutrons, with electrons orbiting around it. At its core, chemistry is the science th studies the motion of these electrons in the field of nuclei and the influence of the electrons on nuclear motion.

Any chemistry taking place between two or more atoms to form molecules, or between molecules themselves, involves the movement of electrons. Electrons $\mathrm{can}$ be shared between molecules or atoms and they can be extracted or added to molecule or atom. Regardless, anytime chemical reaction takes place, electrons move and rearrange, and the specific ways in which that process happens defines the chemistry we observe and use-it determines the behaviour of

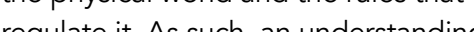
of electron motion in the field of nucle is crucial for our understanding of chemistry, and of the world.

\section{THE QUANTUM REALM} Over the years, there have been several Orocts Because enctrons a vectron motion. move very fast, it is not only difficult (impossible) to observe them directly but their motion also stops obeying the Newtonian physics of the macroscopic world we are used to. When dealing with electronic motion, we enter the realm of quantum dynamics, where electrons can be seen to adopt both particle and wave characteristics, a phenomenon known as 'wave-particle duality'. In fact, in terms of quantum dynamics, electrons are best described by a wave function, which is a mathematical description of electron distribution and thus the energy within a given system.

\section{SCHRÖDINGER'S ERA}

The wave functions that describe crons within a given system appear This equation, first published by Enwin Schrödinger in 1926, is currently the most widely used theoretical model of electronic structure and motion that best accounts for experimental observation, when relativistic effects are not significant. The currently known solutions to the Schrödinger equation have revealed that electrons do not orbit around the atom's nucleus like planets around the Sun. In fact, there are 'clouds' of electron density which are areas around the nucleus where there some probability of finding an electron.

These 'clouds' of electron density probability, which can be described in
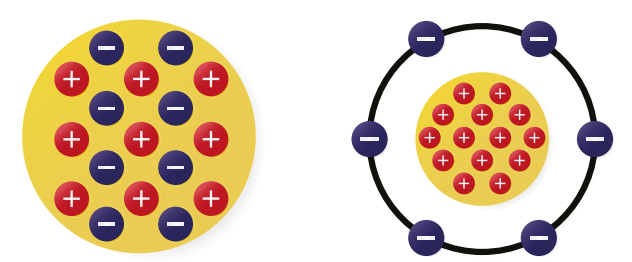

1904 J.J.THOMSON

1803 JOHN DALTON

ctron's location

as we can know of an electron's location,
but even this apparently vague piece of but even this apparently vague piece of
information has already unlocked many information has already unlocked many
further developments. The Schrödinger equation opened the doors to a new era of physics and allowed for mathematical processes, giving rise to the field of 'quantum chemistry' It is on developing and improving quantum chemical models that Professor Weniian Li's's work at the Qingdao Institute for Theoretical and Computational Sciences of Shandong University (China) is vitally focused.

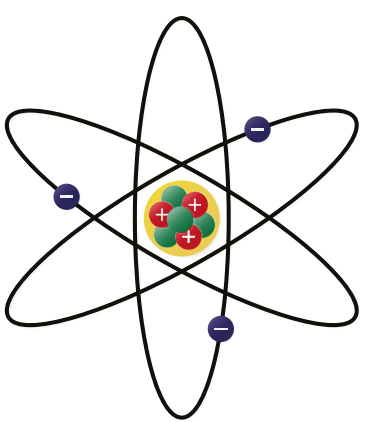

1913

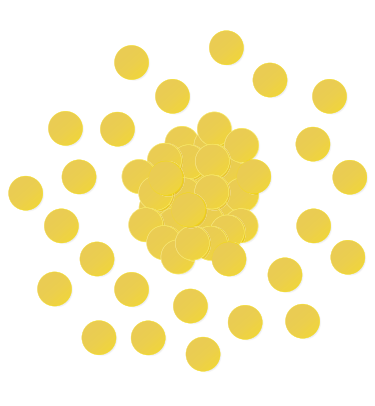

1926 ERWIN SCHRÖDINGER
The first challenge is, of course, to achieve we can attempt to reach the solution for
the whole system - a clear impossibility. an initial guess that is good enough to This difficulty in accounting for electronic lead the model towards the most accurate correlation is one of the main challenges result. This is done by identifying and of electronic structure theory, and there are currently many different address it.

\section{OPTIMISING GUESSES}

In order to address the electron correlation generally attempt to make an initial based on what is known from the exact selecting the orbitals (one-electron wave functions) hat are indispensable to the orbitals have a biven system. These of the final solution and therefor needa precise treatment: the collection of these key orbitals defines an 'active space' of many-electron functions. All other orbitals are accounted for with more approximate calculations, since they do not impact

$\begin{array}{lll}\text { THE LIMITS } & \text { A detailed understanding of electron } & \begin{array}{l}\text { the final result } \\ \text { drastically. }\end{array} \\ \text { OF THE } & \end{array}$

\section{EQUATION} motion is crucial for our understanding

It is precisely by

The Schrödinger of chemistry, and of the world.

optimising the criteri for the selection of

be solven this 'active space be solmplexacly for the hydrogen atom, solutions of the hydrogen atom. Once the that the work of Professor Liu and his the simplest of atoms, containing only initial guess is established, computational collaborators, Mr Zhang from Peking one electron and one proton. In this case, solving the Schrodinger equation inolves simply modelling the interaction proton, which can be considered to be at rest, and a lighter electron that moves around it extremely fast, its motion filling the electron density cloud defined by the corresponding wave function. The Schrödinger equation can be solved exactly for this electron-proton interaction and, as such, an exact description of the electronic structure and energy of the hydrogen atom can be achieved.

However, as soon as a second electron is added to the problem, the situation is complicated beyond an exact solution. The problem is that defining the wave function for one electron depends on the characteristics or the other, and vice-versa. As such, a solution we do not methods will then iteratively optimise
the parameters of the guess until the Hoffmann from the University of North most accurate description possible of the Dakota (USA), is improving quantum

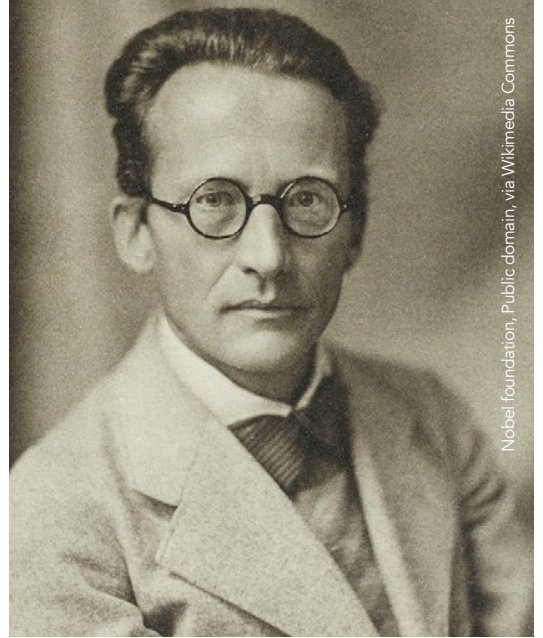

Erwin Rudolf Josef Alex
12 August 1887 . ad previously reported on a computational method called 'iterative and second-order perturbation theory (iCIPT2), which had already proved to be effective in providing near-exact solutions of arbitrarily complex systems at reasonable computational expense. In particular, iCIPT2 is targeted at modelling strongly correlated systems for which static and dynamic correlations are strongly entangled and interchangeable, which is particularly challenging to model. The iCIPT2 method achieves this not only by careful selection of which trial manybut it also uses advaced into account, algorithms that allow for near-exact 


\section{○ 0 \\ Hydrogen Wave Function \\ 8 3. $(0)$ -

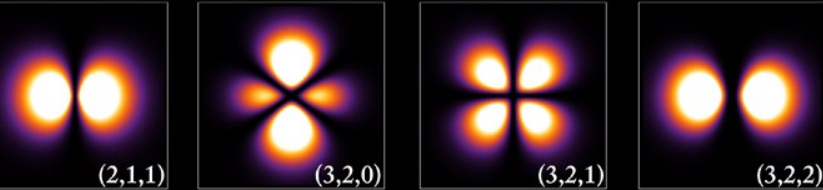 \\ (2) (ब) $(\omega)$ के \\ (cos) * * $*$ \% $\bullet$}

Wave functions of the electron in a hydrogen atom at different energy levels. Quantum mechar ics
cannot predict the exact location of a particle in space, only the probability of finding it at

parameter that allows all unimporant

functions to be untouched throughout

the process. Their efforts made the

iCIPT2 twenty times more efficient than

its original version, with the method also

performing well in terms of accurately

modelling benchmark strongly

correlated systems such as the chromium

dimer, $\mathrm{Cr}_{2}$. As such, the ICIPT2 reveals

issel as a computational method that

ca provide highly accurate modelling

of challenging chemical systems without

computational resources.

The development of new and improved quantum chemical methods, as

undertaken by Mr Zhang and Professors

Liu and Hoffmann, is crucial for future

advances in chemistry. Apart from

unexplored chemical systems, quantum

solutions for the electronic structure of these systems.

MORE FOR NOT MUCH MORE Despite the improvement in active space selection criteria, it is important to bear in mind the computational expense that the final selection requires. Technically, the most accurate solutions would be achieved if all possible trial functions for a system were solved exactly, cut this would lead to impracicable Despite a carefully chosen active space, as becomes possible with advanced methods such as Zhang Liu, and Hoffmann's iCIPT2, quantum chemical calculations can quickly become

expensive even for small increments in

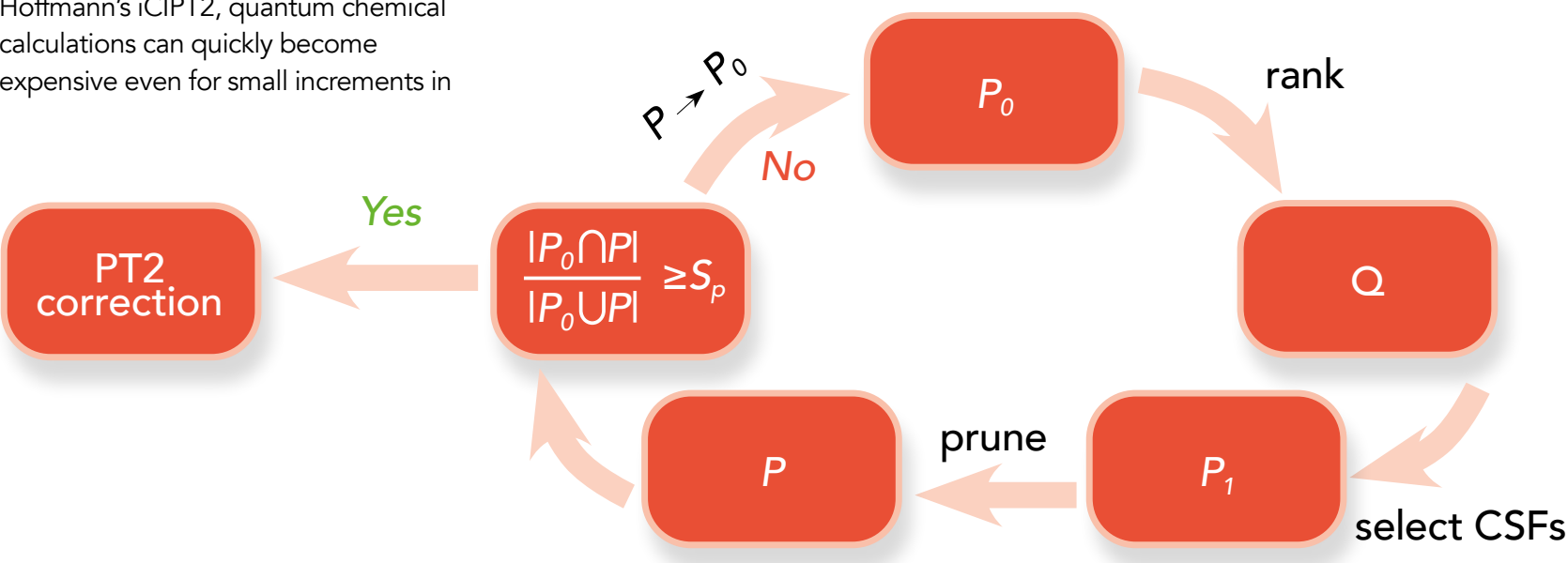

The Schrödinger equation opened the doors to a new era of physics and allowed for mathematical predictions of chemical systems.

complexity. For example, in order to chemistry is also extremely useful in simulate the simplest system used for helping experimentalists make sense modelling biologically important iron- of their observations. Thus, quantum duster [ $\mathrm{Fe}_{\mathrm{S}} \mathrm{S}_{2} \mathrm{CH} \mathrm{H}_{12}{ }^{2}$ it is necessany to include at least 240,374,016 different more expensive and time-consuming functions in the active space. trial-and-error approaches.

\section{Research Objectives}

Professor Weniian Liu and collaborators have developed a quantu electron correlation.

\section{Detail}

\section{Address}

Room 314, Building K1

Shandong University

Qingdao 266237, China

Bio

Chair professor at Shandong University; member of
International Academy of Quantum Molecular Scien (IAQMS), fellow of Royal Society of Chemistry as well as Asia-Pacific Association of Theoretical and Computational Chemists (APATCC); recipient of Bessel Award of Humbold Foundation, Annual Medal of IAOMS, Pople and Fuku Medals of APATCC.

Funding

(Grant Nos 21833001 and 21973054) and University of North Dakota

\section{Collaborator}

Mr Ning Zhang and Professor Mark R. Hoffman CO 政 $\underbrace{}_{\text {Palladium }}$

References

Lucibella, Michael (2015). This Month in Physics History: April 28, 1926: Schroedinger Describes "Wave Mechanics" in Letter to Einstein. Tonlinej APS News. Available at. https./Mww aps.org/publications/apsnews/201504/physicshistory.cfm

Liu, Wenjian and Hoffmann, Mark R. (2016). iCl: Iterative toward full Cl. J Chem Theory Comput 12, 1169-1178

Zhang, Ning, et al (2020). Iterative Configuration Interaction with Selection. J Chem Theory Comput, 16, 2296-2316

Zhang, Ning, et al (2021). Further development of iCIPT2 for Strongly Correlated Electrons. J Chem Theory Comput 17, 949-964

Personal Response

What is the most challenging part of developing these

II Identifying and selecting a priori the most important configurations as well as fast evaluation of their matrix

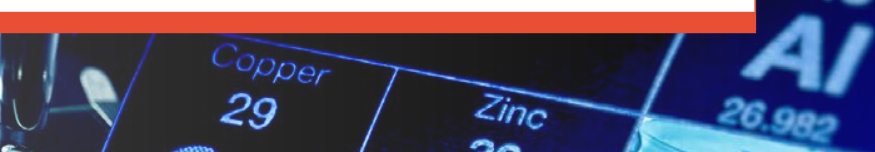

- $\mathrm{Cu}_{63.54}^{29} \mathrm{Zn}^{30}$
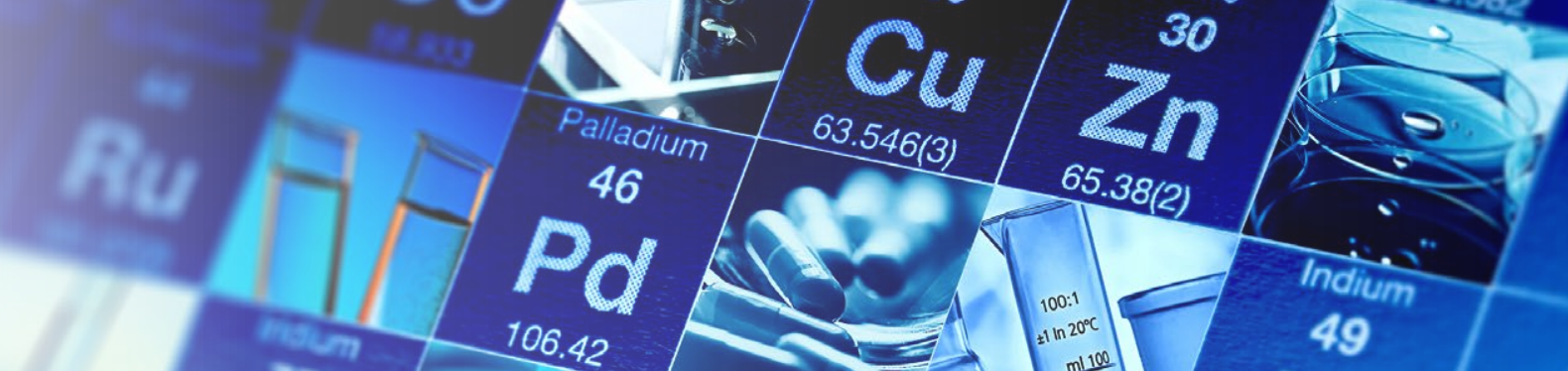

Inclium

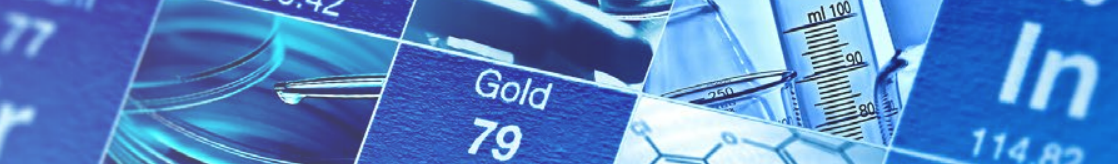

ln

44

$\mathrm{Sn}^{2}$

109

$M i$
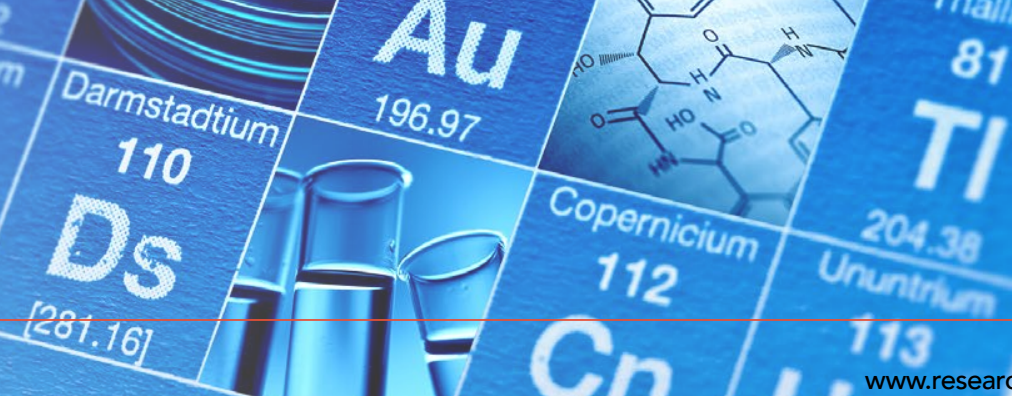

81 